\title{
Striatal outcome processing in healthy aging
}

\author{
KARIN M. CoX \\ University of Pittsburgh, Pittsburgh, Pennsylvania \\ and Center for the Neural Basis of Cognition, Pittsburgh, Pennsylvania \\ Howard J. Aizenstein \\ University of Pittsburgh, Pittsburgh, Pennsylvania \\ University of Pittsburgh School of Medicine, Pittsburgh, Pennsylvania \\ and Center for the Neural Basis of Cognition, Pittsburgh, Pennsylvania \\ AND \\ JuLie A. Fiez \\ University of Pittsburgh, Pittsburgh, Pennsylvania \\ and Center for the Neural Basis of Cognition, Pittsburgh, Pennsylvania
}

\begin{abstract}
Functional MRI of young adults has implicated the striatum in the processing of rewarding and punishing events. To date, only two published experiments (Samanez-Larkin et al., 2007; Schott et al., 2007) have explored similar phenomena in older adults, with both studies emphasizing the anticipation of monetary outcomes. To better understand older participants' striatal responses to delivered outcomes, we engaged 20 older adults and 13 younger adults in a card-guessing task that rewarded correct guesses with monetary gain and punished incorrect guesses with monetary loss. Overall, the older adults retained most of the typical features of the striatal response, so that activity in the caudate head showed reliable differentiation between rewards and punishments during the 6 - to 9 -sec postoutcome window. Comparison of the older and younger adults also pointed to some potential aging effects on outcome activity, including reductions in the magnitude and extent of striatal activation, and a trend for the older adults to show a decreased early punishment response. In sum, our data suggest that the signaling of outcome valence remains relatively stable into late adulthood, although more research is needed to understand some subtle changes that might occur across the life span.
\end{abstract}

Evidence from animals and humans has established the striatum as a key structure for processing motivationally relevant events. In rodents and primates, both appetitive and aversive stimuli modulate firing patterns in the striatum (Ravel, Legallet, \& Apicella, 2003; Roitman, Wheeler, \& Carelli, 2005; Schultz, Tremblay, \& Hollerman, 2000; Williams \& Eskandar, 2006) and in the dopamine-producing neurons that project to it (e.g., Hollerman \& Schultz, 1998; Ungless, Magill, \& Bolam, 2004). Likewise, humans in functional MRI experiments have shown striatal activation to a range of motivating outcomes (e.g., Berns, McClure, Pagnoni, \& Montague, 2001, for juice; Delgado, Nystrom, Fissell, Noll, \& Fiez, 2000, for money), as well as to outcome-predictive cues (e.g., Knutson, Westdorp, Kaiser, $\&$ Hommer, 2000; Wittmann et al., 2005). From a computational standpoint, these responses are often described as signaling an error in reward prediction (e.g., O'Doherty et al., 2004), so that the greatest striatal activity is driven by the least predictable cues and outcomes. This predictiondriven response pattern is assumed to reflect the involvement of the striatum in reinforcement-based learning, an assumption that is supported by reports of altered learning behavior when striatal dopamine is manipulated (e.g., Pessiglione, Seymour, Flandin, Dolan, \& Frith, 2006).

Striatal reward- and punishment-signaling has been well documented in young adults, but the anatomical and behavioral correlates of aging call into question the stability of these phenomena across the life span. At the neuroanatomical level, declines in older adults' striatal volume (Raz, Torres, \& Acker, 1995) and in dopamine D1 and D2 receptor availability (Volkow et al., 1998; Wang et al., 1998) suggest that the learning and motivational functions of the striatum may be affected as well. Similarly, at the behavioral level, altered striatal function may help explain why older adults have shown learning deficits on tasks that require the processing of positive and negative outcomes (e.g., Denburg, Recknor, Bechara, \& Tranel, 2006; Marschner et al., 2005; Mell et al., 2005; Weiler, Bellebaum, \& Daum, 2008). Thus, there are compelling reasons to expect that reward and punishment processing might be disrupted in the aging striatum. Efforts to address this question with neuroimaging have just begun to emerge.

To date, two fMRI experiments have focused on reward and punishment activity in older adults (Samanez-Larkin

K. M. Cox, kmc51@pitt.edu 
et al., 2007; Schott et al., 2007); in each case, the researchers used customized versions of the monetary incentive delay (MID) task (Knutson et al., 2000). The MID task structure can be illustrated by the recent design implemented by Schott et al., who probed for older and younger adults' activation to reward-predictive cues. In Schott et al.'s paradigm, symbolic cues indicated the potential earnings on each trial of a numerical judgment task. On trials initiated by neutral cues, participants did not receive any rewards or penalties for their task performance, but reward cues predicted monetary gain for a successful response and monetary loss for a slow or inaccurate response. Individually calibrated response deadlines ensured that success rates were generally high ( $~ 80 \%$ of trials) across participants and conditions.

Schott et al. (2007) used this version of the MID task to determine whether the older and younger participants would acquire striatal responses to the reward-predictive cues. To promote the development of cue-reward associations, a prescan practice session exposed the participants to the individual cues and their relationship with the different possible outcomes. During the subsequent imaging session, the two age groups showed highly divergent patterns of striatal activity: Although the reward cues triggered robust anticipatory responses in the younger adults, these cues failed to elicit activation in the older adult group. In interpreting this age difference, Schott et al. proposed that the disappearance of anticipatory signals in the older adults was closely related to the reward-learning deficits that have been described in the behavioral literature (e.g., Marschner et al., 2005; Mell et al., 2005). Consistent with this interpretation, only the older adults showed activity associated with reward delivery, as would be expected if these participants did not effectively predict reward likelihood on the basis of the cues.

A study by Samanez-Larkin et al. (2007) also examined anticipatory signaling in aging, with an interest in the neural responses to both reward- and punishment-predictive cues. In the context of a buttonpress task, the authors presented cues that stated either the gain $(\$ 0.00, \$ 0.50$, or $\$ 5.00)$ that could be earned or the loss $(\$ 0.00, \$ 0.50$, or $\$ 5.00)$ that could be avoided by performing successfully ( $66 \%$ of trials). The use of explicit cues may have minimized the need to learn cue-outcome associations; on the gain-framed trials, both older and younger adults showed reliable striatal responsiveness to the cued value. In contrast, the loss-framed trials revealed a substantial group difference in the response to prospective punishment: Whereas the magnitude of the loss cues modulated striatal activity in the younger participants, reliable magnitude effects were absent in the older ones. Such decreased sensitivity to anticipated loss is consistent with the idea that a "positivity bias" governs older adults' processing of affective information, as has been shown to be the case for other kinds of emotionally salient stimuli (see Mather \& Carstensen, 2005, for a review). It is notable, however, that Samanez-Larkin et al. did not find similar biases during the actual experience of negative outcomes; both age groups showed significant deactivations to loss delivery (and also to reward omission, during the gain-framed trials).

In summary, both the Samanez-Larkin et al. (2007) and the Schott et al. (2007) studies emphasized age-related changes in incentive anticipation, and they provided initial evidence that the aging striatum retains the capacity to signal reward and punishment outcomes. These studies also leave open several questions for further exploration, including some remaining uncertainty regarding older adults' outcome processing. The outcome phase data provided by both Samanez-Larkin et al. and Schott et al. were somewhat limited. For example, in the Schott et al. study, no hemodynamic time-course estimates were presented, punishment-related responses were not analyzed, and the lack of outcome responsiveness in the younger adults precluded a meaningful age group comparison. In the Samanez-Larkin et al. study, the outcome phase analyses were somewhat more complete, but were subject to some other concerns. In both the older and younger participants, outcome-related activity did not reach the rigorous statistical threshold that was surpassed by the cue responses, and no outcome signals were found in the region associated with the authors' loss-anticipation findings (i.e., a focused medial caudate volume of interest). Additionally, although Samanez-Larkin et al. did present striatal time courses, the interpretation of their data may be complicated by the fast event-related design from which they were derived; that is, in the presented time courses, the temporal profile of the outcome response may be obscured by activation related to the initiation of the next trial.

In the present research, we aimed to provide more robust estimates of older adults' outcome signals. Specifically, our first objective was to determine whether major features of striatal outcome processing are preserved in healthy aging, particularly when the context is known to elicit reliable outcome signaling in the young. The importance of this goal is underscored by the evidence for learning-related and neuroanatomical changes in older adults, since these factors suggest that the stability of the striatal response cannot be taken for granted. Additionally, a thorough description of healthy older adults' outcome activity could serve as a useful baseline for future studies of basal ganglia dysfunction; indeed, one of the original motivations for the present work was to support a longer term research agenda in patients with early stage Parkinson's disease.

A second objective, which was largely motivated by the emerging reports of anticipatory differences in aging, was to continue to explore the possibility of age-related differences in striatal outcome signaling. Samanez-Larkin et al. (2007) did not report major aging effects on outcome activity, but trends in the broader literature suggest that this question may merit additional attention. For example, in a recent eventrelated potential (ERP) study, Eppinger, Kray, Mock, and Mecklinger (2008) demonstrated a significant reduction in older adults' feedback-related negativity during a probabilistic learning task. Although this electrophysiological finding does not have direct implications for striatal function, it does provide evidence that affective biases may apply to the outcome phase, and thus encourages further efforts to identify age group differences in striatal outcome activity.

To acquire strong estimates of outcome responding in aging, we used a well-established card-guessing task that was developed in our laboratory (Delgado et al., 2000). On each trial of the guessing task, participants were instructed to guess the value of a concealed playing card 


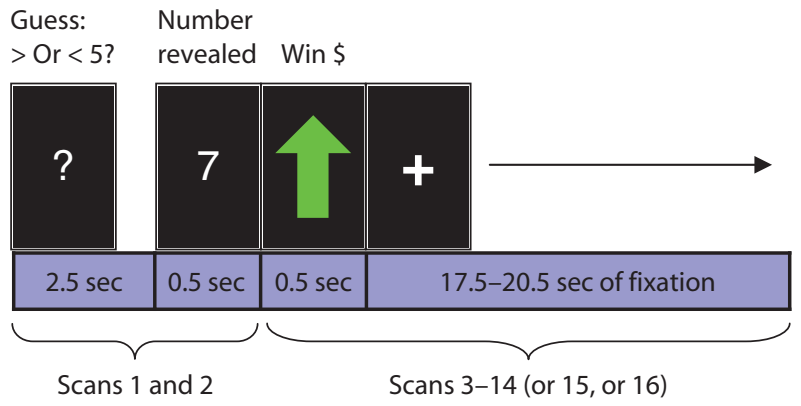

$\mathrm{TR}=1.5 \mathrm{sec}$

Figure 1. The modified version of the card-guessing task, with a lengthened intertrial interval to account for the possibility of prolonged hemodynamic responses in older participants. The figure depicts a reward trial. During punishment trials, a red, downward-pointing arrow was displayed.

(Figure 1). Each guess was followed by the presentation of either a green arrow, indicating a correct guess and a small monetary reward, or a red arrow, indicating an incorrect guess and a small monetary loss.
Three features of the guessing task make it a suitable complement to prior work with the MID paradigm. First, the task design accounts for the importance of unpredictability in driving striatal outcome responses (Berns et al., 2001; Delgado, Miller, Inati, \& Phelps, 2005) by maintaining a maximal level of uncertainty throughout the task. Reward outcomes occur on a predetermined $50 \%$ of trials, thereby preventing participants from learning any strategies to improve their performance. This balanced rewardto-punishment ratio contrasts with the typical design of the MID task, in which hit rates are generally maintained above 50\% (e.g., Knutson et al., 2000; Wittmann et al., 2005). Second, the guessing task has been shown to generate highly reproducible outcome effects, such that wholebrain analyses have consistently identified a focused caudate region that discriminates between the reward and punishment outcomes. The reliability of this focus can be illustrated by the combined activation maps from our previous work (Delgado, Locke, Stenger, \& Fiez, 2003; Delgado et al., 2000; Delgado, Stenger, \& Fiez, 2004; Tricomi, Delgado, \& Fiez, 2004; Tricomi, Delgado, McCandliss, McClelland, \& Fiez, 2006). The maps indicate a shared region of outcome sensitivity in the caudate head (i.e., within

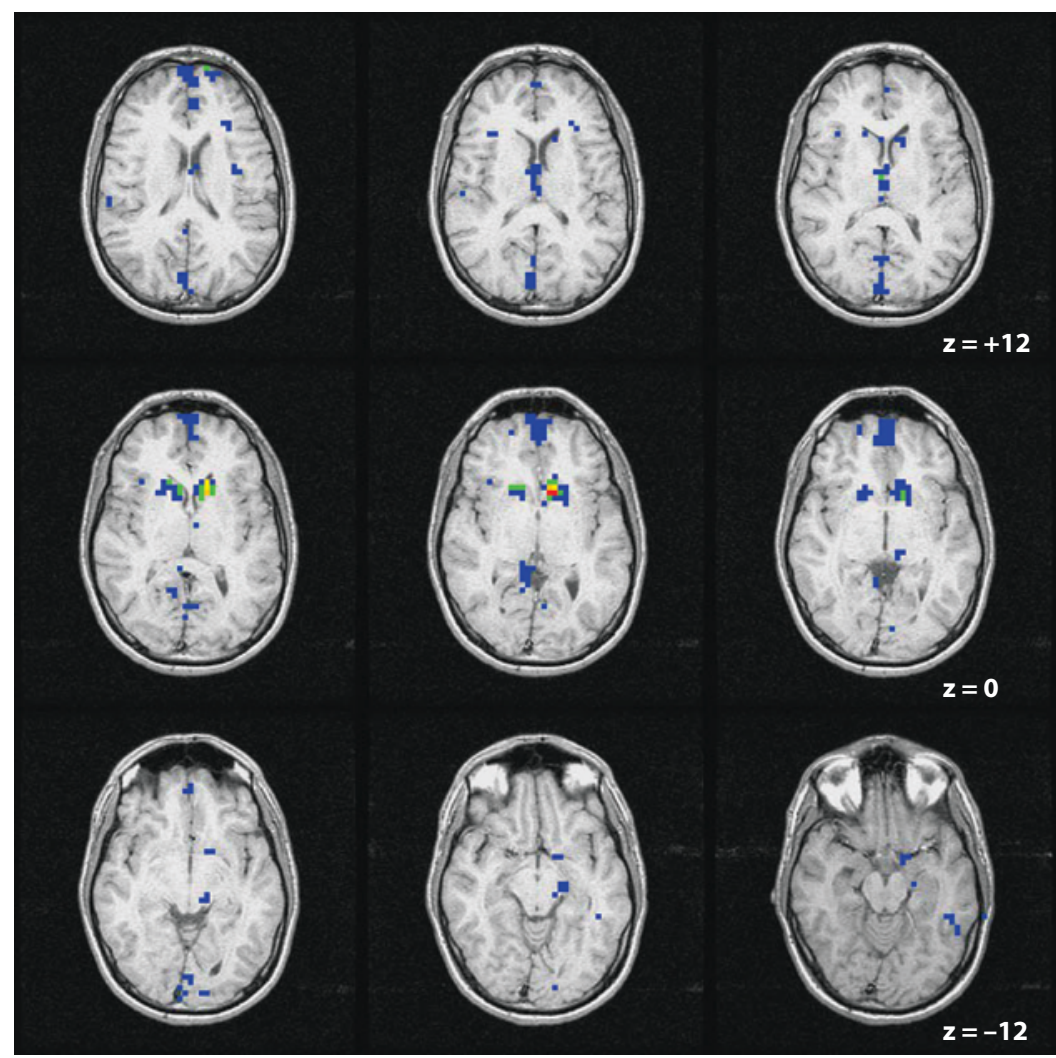

Figure 2. Overlay of the outcome-sensitive activity found in five young adult studies using the card-guessing task (Delgado et al., 2003, Experiment 2; Delgado et al., 2000; Delgado et al., 2004, high-incentive trials; Tricomi et al., 2004, Experiment 3, choice condition; Tricomi et al., 2006). Each study contributed a statistical map of the valence $\times$ time interaction, thresholded at $p<.001$ with a contiguity requirement $\geq 3$ voxels. Maps were overlaid on the reference brain used for three of the studies (Delgado et al., 2003; Delgado et al., 2000; Delgado et al., 2004); data that were not originally presented in this space (Tricomi et al., 2004; Tricomi et al., 2006) were aligned to the reference with a linear model (Woods, Grafton, Watson, et al., 1998). Colors indicate the number of studies in which a voxel showed a significant effect: Blue $=1$, green $=2$, yellow $=3$, orange $=4$, red $=5$. Images are shown according to radiological convention (right $=$ left). 
a limited area near the anterior commissure, as shown in Figure 2). Convergent fMRI data from a variety of instrumental learning scenarios (e.g., Haruno \& Kawato, 2006; Nieuwenhuis et al., 2005; O'Doherty et al., 2004) have implicated a similar striatal subregion in outcome processing. Third, the slow-event related design of the guessing task has permitted a detailed temporal characterization of the striatal response. Specifically, a reward tends to evoke a sustained hemodynamic response characterized by a relatively slow return to baseline; a punishment generally results in a rapid, high-amplitude response peak followed by a decrease in the signal below baseline levels.

To determine whether similar response patterns are retained in healthy aging, we analyzed data from 20 older adults and 13 younger adults who had completed our guessing task protocol. In accordance with the primary goal of the study, initial analyses were aimed at characterizing the older adult response, with a specific focus on whether or not these participants would retain the major response features described in previous research. Subsequent analyses involved direct comparisons of older and younger adults' outcome activity, since recent research has suggested potential differences in these groups' processing of motivationally relevant stimuli.

\section{METHOD}

\section{Participants}

Healthy right-handed older adults (age range $=50-70$ years) and younger adults (age range $=18-35$ years) were recruited with flyers posted around the University of Pittsburgh campus and the surrounding community, and with advertisements placed in newsletters targeted toward a senior audience. Older participants were also recruited from the registry maintained by the University of Pittsburgh Alzheimer's Disease Research Center. Participants were required to be generally healthy and were excluded from participation if they reported any history of illicit drug use, stroke, or a major neurological or psychiatric disorder, or if they were currently taking any psychotropic medications. Individuals who satisfied the inclusion and exclusion criteria were further screened for depression and general cognitive impairment; all participants considered for analysis scored $\leq 13$ on the Beck Depression Inventory (BDI-II; Beck, Steer, \& Brown, 1996) and $>26$ on the Mini-Mental State Examination (Folstein, Folstein, \& McHugh, 1975).

In light of our longer term interest in early stage Parkinson's disease, the 50- to 70-year age range defined for the older group is consistent with the typical demographics reported in studies of patients with Parkinson's (e.g., Swainson et al., 2006). The lower bound of this range includes participants who may not be traditionally classified as "elderly," but it is likely that meaningful age-related changes will have already occurred by this point in life. Linear declines in the relevant anatomical markers have been reported in samples of $<70$ years of age: Hasan, Halphen, Boska, and Narayana (2008) reported age-correlated reductions in caudate volume (age range $=$ 19-59 years), and Bäckman et al. (2000) found age-related declines in striatal D2 receptor binding (age range $=21-68$ years). Important behavioral changes have also been noted in late middle age. For example, in a study of self-reported affect (Carstensen, Pasupathi, Mayr, \& Nesselroade, 2000), the frequency of negative emotion declined until approximately 60 years of age and remained relatively constant into old age. Thus, the literature on aging provides some support for the assumption that our groups differed along dimensions that may be relevant for striatal function.

Twenty-nine older adults and 21 younger adults were enrolled in the study. Informed consent and experimental procedures were conducted in accordance with the regulations set by the University of Pittsburgh Institutional Review Board. Five participants (3 older, 2 younger) did not complete the imaging protocol due to either technical difficulties or general discomfort in the scanner environment. Data from 4 of the participants who completed the experiment were removed due to either participant drowsiness ( 2 younger, 1 older) or the discovery of a scanner-related artifact (1 older). Excessive head motion (i.e., displacement greater than $4 \mathrm{~mm}$ or $4^{\circ}$ ) resulted in the removal of 6 additional subjects ( 3 younger, 3 older). The final analyzed sample included 20 older adults $(7$ males, age range $=$ $51-68$ years, $M=57.60, S D=4.63$ ) and 13 younger adults ( 6 males, age range $=18-28$ years, $M=22.31, S D=3.28$ ). For 2 older participants with movement spikes isolated to the final run of scanning, the initial portion of the functional data set (i.e., 84 trials of the guessing task) was retained for analysis. For an additional 2 older adults and 1 younger adult, early termination of the scanning session (due to technical problems or participant discomfort) resulted in the loss of the last one or two runs of functional acquisition (i.e., 12 or 24 trials of the guessing task).

\section{Behavioral Task}

During the scanning session, participants completed eight runs of the card-guessing task, with 12 trials per run. The task was programmed using E-Prime software (Schneider, Eschman, \& Zuccolotto, 2002) and was presented in the scanner environment using the Integrated Functional Imaging System (IFIS-SA; Invivo, Gainesville, FL). Participants made their responses with the indexand middle-finger buttons of a response glove on their right hand. Response and reaction time (RT) data were recorded for each trial. Trials on which no response was detected were omitted from subsequent analysis. Due to a technical problem with the glove unit, responses were not registered for a small portion of trials for 3 of the younger participants; the remaining data from these participants were retained. After accounting for missed trials and removed runs (see above), the older and younger participants contributed a comparable number of trials to analysis $(M=92.70, S D=5.62$ trials for the older adults; $M=91.15, S D=6.16$ trials for the younger adults).

As in previous work (e.g., Delgado et al., 2000), the temporal structure of the guessing task was based on a slow-event related design. Since some studies of older adults' BOLD signal have reported a relatively slow decay to baseline (Aizenstein et al., 2004; Richter $\&$ Richter, 2003), we modified the original parameters of the task to account for this possibility in our participants. Whereas the timing of the task events remained the same, we increased the intertrial interval by several seconds. A trial began with the presentation of a concealed playing card for $2.5 \mathrm{sec}$ (Figure 1); during this time, participants used a buttonpress to guess whether the card value was greater than or less than 5 . Following this choice period, the number was revealed for $0.5 \mathrm{sec}$, followed by a 0.5 -sec display consisting of an upward-pointing green arrow (correct guesses) or a downwardpointing red arrow (incorrect guesses). If the participant failed to respond, the choice period was followed by the 1-sec display of a pound sign (\#). Each trial ended with a fixation period of at least $17.5 \mathrm{sec}$; research on the BOLD signal in aging suggests that this interval should be sufficient for responses to return to baseline (Richter \& Richter, 2003). However, the possibility of longer response durations - which would invalidate the typical trial-averaging approach to response estimation - could not be ruled out. Therefore, as a precaution, an additional $1.5-3.0 \mathrm{sec}$ of fixation were added to a random subset of trials. In the event that activity persisted beyond the minimum 17.5-sec fixation period, this extra jitter would allow responses to be estimated with a GLM-based deconvolution program (e.g., AFNI 3dDeconvolve; Ward, 2001).

All participants read written instructions for the guessing task and received 12 trials of practice before entering the scanner. They were told that for each correct guess, they would earn $\$ 4$, and for each incorrect guess, they would lose $\$ 2$. The 2:1 gain-to-loss ratio is typical for this task and was motivated by classic decision-making research showing a disproportionate influence of monetary loss on 
choice behavior (e.g., Tversky \& Kahneman, 1981). Unbeknownst to the participants, outcomes were delivered according to a fixed, predetermined sequence, so that $50 \%$ of the guesses were correct and $50 \%$ were incorrect. No rewards or penalties were delivered for missed trials. At the end of the session, the participants were debriefed about the nature of the task and were paid $\$ 80$ for their participation in the study.

\section{fMRI Data Acquisition}

All participants were scanned at the UPMC Presbyterian Hospital MR Center with a 1.5T GE Signa whole-body scanner. Each 75-min scanning session began with the acquisition of structural data. For purposes of intersubject registration, we collected high-resolution whole-brain structural spoiled gradient-recalled data (SPGRs), as well as lower resolution structural images. SPGRs were acquired in 124 axial slices with an in-plane resolution of $0.94 \mathrm{~mm}^{2}$ and a voxel depth of $1.50 \mathrm{~mm}$. T1-weighted structural in-planes were acquired in 40 contiguous oblique slices parallel to the AC-PC line, with an inplane resolution of $0.94 \mathrm{~mm}^{2}$ and a voxel depth of $3.80 \mathrm{~mm}$. Functional data were collected in 20 oblique slices (resolution $=3.75 \times$ $3.75 \times 3.80 \mathrm{~mm}$ ), using a one-shot forward spiral pulse sequence $\left(\mathrm{TR}=1,500 \mathrm{msec}, \mathrm{TE}=35 \mathrm{msec}, \mathrm{FOV}=24 \mathrm{~cm}\right.$, flip angle $\left.=70^{\circ}\right)$. Functional slices overlapped with a subset of the structural in-planes and included 15 slices superior and 5 slices inferior to the AC-PC line. This slice prescription allowed for coverage of the dorsal and ventral striatum in all of the participants, as well as a large portion of the cortex and the most superior regions of the cerebellum. Functional coverage did not include some dorsal regions of the frontal and parietal cortex, some ventral regions of the frontal and temporal cortex, and the majority of the brainstem and the cerebellum.

The participants completed 96 trials of the guessing task across eight runs of functional acquisition, with each run lasting approximately $4.6 \mathrm{~min}$. As part of the same experimental session, both participant groups also completed two runs of a bimanual finger-tapping task; the order of the card-guessing and finger-tapping tasks was counterbalanced across participants. Data from the finger-tapping task were collected in order to support future analyses in the sensorimotor striatum and are not reported here.

\section{Preprocessing of Functional Data}

The raw data were preprocessed using the NeuroImaging Software package developed at the University of Pittsburgh and Princeton University (NIS-3.6) and Automated Image Registration (AIR 3.08; Woods, Grafton, Holmes, Cherry, \& Mazziotta, 1998). Functional images were corrected for participant movement and between-run differences in baseline intensity. To account for gradual drift in the scanner signal, voxelwise linear trends were estimated and removed within each run.

In preparation for group-level analyses, the preprocessed data were spatially normalized to a reference image acquired from a 29-year-old male participant who had not otherwise contributed to the present data set. Our past research relied on an affine model to perform this coregistration step (Woods, Grafton, Watson, Sicotte, \& Mazziotta, 1998), but the well-documented atrophy of cortical (Raz et al., 2004) and subcortical (Raz et al., 1995) regions in aging motivated the use of a higher dimensional approach. Therefore, in the present study, each participant's SPGR image was warped to the reference brain according to a fully deformable coregistration procedure, ${ }^{1}$ using free software available through the National Library of Medicine's Insight Segmentation and Registration Toolkit (ITK; www.itk.org). In recent comparative studies (Wu, Carmichael, Lopez-Garcia, Carter, \& Aizenstein, 2006; Wu, Mazurkewicz, Nable, \& Aizenstein, 2006), the fully deformable model produced superior intersubject alignment relative to that produced by nonlinear methods with fewer free parameters - that is, those provided by AIR (Woods, Grafton, Watson, et al., 1998) and Statistical Parametric Mapping (Friston et al., 1995). Thus, we expected that the fully deformable method would also improve coregistration among our older and younger participants, although it is acknowledged that better alignment does not eliminate all methodological concerns associated with structural atrophy.

Each warped SPGR image was viewed to confirm that no serious errors in registration had occurred (e.g., displacement or gross distortion of the anatomy). The warps defined for the structural images were then applied to the functional data sets, which had been upsampled to a voxel size of $1 \mathrm{~mm}^{3}$. Prior to statistical analysis, warped functional images were downsampled to their original resolution. For each downsampled volume, intensity values were normalized to a global mean of 2,000 and spatially smoothed with a threedimensional Gaussian filter (4 mm FWHM).

\section{Statistical Analysis of Functional Data}

Based on observations that hemodynamic responses in the striatum did appear to resolve to baseline within a 17.5 -sec fixation period, the time-course estimates reported for the present experiment reflect standard trial-based averaging. ${ }^{2}$ Therefore, for each voxel and each participant, average intensity values were computed for the first $21 \mathrm{sec}$ (or, with a TR of $1.5 \mathrm{sec}$, the first 14 scans) of the reward and punishment trials. Participant-level averages were then submitted to group-level statistical analysis.

Group-level analyses were performed with the NIS package, AFNI (Cox, 1996), and SPSS for Windows (Release 15.0.0, SPSS, Inc., Chicago). Two kinds of masks were prepared for the voxelwise tests: a whole-brain mask (for exploratory full-brain analyses) and a striatum mask (for a priori tests of striatal activity). At the whole-brain level, the AFNI 3dAutomask program was applied to each participant in order to create a functional mask restricted to voxels of suprathreshold intensity. The individual masks were then combined into a group-level mask that included only those voxels that were represented in each participant. Since signal dropout, individual differences in brain size, and image warping can alter the effective extent of participants' images, the whole-brain mask included only 18 of the original 20 functional slices. For the striatum mask, the left and right caudate (head and body) and putamen were manually traced on the reference brain. Any striatal voxels that were not present in the whole-brain mask (including the entire nucleus accumbens) were similarly excluded from the striatum mask.

Our masking procedure did exclude a small region of the ventral caudate head (see mask outline, Figures 3 and 5); this signal loss is consistent with previous quantitative reports of susceptibility artifacts in the striatum (Lipschutz, Friston, Ashburner, Turner, \& Price, 2001). Inspection of the single-participant masks revealed considerable variability in the severity of striatal signal loss, particularly within the slice immediately ventral to the AC-PC line. A large portion of participants did have suprathreshold striatal signals in this region $\left(n_{\text {older }}=14, n_{\text {younger }}=12\right)$. Thus, we replicated our voxelwise analyses in this subgroup, in order to determine whether any previously undetected effects would emerge in the ventral striatum. These exploratory analyses did not reveal any ventral striatal activity that had not been observed in the full participant sample, with the exception of three additional outcome-sensitive voxels in the younger adult subgroup (which were attached to clusters that were already apparent in the full younger adult sample). Therefore, since the subgroup analyses provided little new information about the ventral striatum, only the results from the entire set of participants are reported here.

Statistical analysis consisted of two stages: analyses specific to the older adults, and comparisons of our older and younger groups. These stages are described in detail below.

\section{Characterization of the Older Adults' Outcome Response}

The initial stage of analysis was aimed at characterizing the older adults' outcome activity and testing for the major response features that have been described in previous younger adult research. Therefore, a voxelwise analysis was used in order to locate the older adults' outcome-sensitive striatal clusters, and hemodynamic re- 
sponses from each cluster were tested for the presence of specific temporal features.

Spatial extent of the older adults' outcome activity. The older adults' functional data were submitted to a two-way repeated measures ANOVA, with valence (reward vs. punishment) and time (an index of the 12 postoutcome scans in each trial, T3-T14) as factors. The principal effect of interest was the valence $\times$ time interaction, which identifies brain regions with differential response profiles for the reward and punishment outcomes. To take advantage of our strong a priori interest in the striatum, results were thresholded at two levels: $p<.001$ (striatum-focused analyses) and $p<.0001$ (exploratory whole-brain analyses). Although the $p<.0001$ threshold has been the typical standard for the guessing task (e.g., Delgado et al., 2000; Tricomi et al., 2004), the $p<.001$ threshold may be useful for exploring the wider extent of striatal activity, particularly in ventral striatal regions (see Delgado et al., 2000). To protect against Type I error, a cluster threshold of 3 contiguous voxels was also applied to the striatum-focused and whole-brain activation maps (Forman et al., 1995; see also Delgado et al., 2000). Thresholded maps were converted into the standard atlas space of Talairach and Tournoux (1988), in order to determine the anatomical focus of each cluster.

Striatal time-course features in the older adults. The older adults' hemodynamic time courses were extracted from the striatal clusters that showed significant valence $\times$ time effects at $p<.001$ (specifically, left and right clusters in the anterior caudate/putamen). Time-course data were examined for the presence of the response features that have been noted in earlier reports of the card-guessing task. Two-tailed $t$ tests were used to compare the reward and punishment signals at three timepoints: T4 (1.5-3.0 sec following outcome onset) and T7 and T8 (6-9 sec following outcome onset). The T4 timepoint was tested to determine whether the punishment response exceeded the reward response during the early postoutcome period, as has been reported previously in younger adults (Delgado et al., 2003). Similarly, tests at T7 and T8 were motivated by studies that have shown a higher amplitude for reward than for punishment activity during this later postoutcome period (Delgado et al., 2003; Tricomi et al., 2004; Tricomi et al., 2006). All $t$ test results were interpreted with a significance threshold of $p<.05$ (Bonferronicorrected for the three separate contrasts, resulting in an effective threshold of $p<.017$ ).

\section{Between-Groups Comparisons}

In the second stage of analysis, the older and younger adults' striatal responses were directly compared. The age group comparison included three steps. First, maps of the two groups' outcome activity were directly overlaid, so that overlapping regions (i.e., conjunction regions) could be identified. Second, between-groups comparisons were performed on the time courses obtained from the conjunction regions. Finally, as a complement to the first two analyses, aging effects on the outcome response were also assessed at the voxelwise level.

Overlay of the older and younger adults' outcome activity. The younger adults' functional data were submitted to a similar voxelwise ANOVA, as was described for the older participants above. As in the analyses of the older adults, the younger adults' results were thresholded at $p<.001$ within the striatum and $p<.0001$ outside of the striatum (the contiguity threshold was 3 voxels for all analyses). The two groups' valence $\times$ time maps were then directly overlaid. The resulting overlap map revealed left and right striatal regions defined by the conjunction of the older and younger adults' activity, as well as voxels that were present only in either the older or the younger participants' map.

Comparison of the older and younger adults' striatal time courses. Comparison of the two groups' time-course data was conducted for both the left and right conjunction regions. This particular approach is useful for considering how age affects the response time courses when reliable outcome sensitivity can be assumed for each group. The conjunction region time courses were assessed using a three-way mixed ANOVA, with age group as a between-participants factor and valence and time as within-participants factors. All 12 postoutcome timepoints (T3-T14) were included in the time factor, because we made no specific prediction of how the older and younger adults' time courses might differ. The principal effect of interest was the three-way group $\times$ valence $\times$ time interaction, which would indicate differential aging effects on the reward and punishment responses. Two-way group $\times$ time results were also noted. A significance threshold of $p<.05$ was applied to the interaction tests.

Voxelwise comparisons of the older and younger adults. A three-way group $\times$ valence $\times$ time ANOVA was also performed on all voxels falling within our functional masks. All 12 postoutcome timepoints (T3-T14) were included in the time factor. Relative to our cluster-based analyses, the advantage of this voxelwise approach is its sensitivity to effects that may not spatially coincide with the older-younger conjunction regions. The voxelwise analyses were used for generating activation maps for the group $\times$ valence $\times$ time and group $\times$ time interactions. As was described for the analyses specific to the older adults, the between-groups voxelwise results were thresholded at $p<.001$ for striatum-focused analyses and $p<$ .0001 for whole-brain analyses, with a contiguity requirement of 3 voxels.

\section{RESULTS}

\section{Behavioral Results}

Older and younger adults' RTs did not significantly differ for the card-guessing task $[M=712.81 \mathrm{msec}, S D=$ 36.46 for the older adults; $M=663.99 \mathrm{msec}, S D=60.68$ for the younger adults; $t(31)=0.73]$. A comparison of the two groups' response patterns found no significant difference in the rates at which each response option was chosen, with both the older and younger participants choosing the "greater than 5" option approximately half of the time [ $56 \%$ in the older group, $51 \%$ in the younger group; $t(31)=1.30]$.

\section{Characterization of the \\ Older Adults' Outcome Response}

Spatial extent of the older adults' outcome activity.

Voxelwise analyses revealed robust valence $\times$ time effects in the older adults' striatum, with reliable activity found at both the $p<.001$ and $p<.0001$ thresholds (Figure 3; Table 1). Within the striatum mask, significant clusters $(p<.001)$ were located in the right caudate head/anterior putamen (peak Talairach coordinates $=13,14,4$; cluster size $=24$ voxels), the left caudate head (peak $=$ $-14,10,8$; size $=16$ voxels), and the left ventral putamen ( peak $=-21,0,-4$; size $=3$ voxels). Qualitative comparison of these clusters with the striatal regions identified in past research (Figure 2) suggests that the typical foci of outcome sensitivity were generally preserved in the older adults. The most robust effects fell within the caudate head in the first one or two slices dorsal to the slice containing the anterior commissure.

Beyond our a priori focus on the striatal nuclei, significant valence $\times$ time effects $(p<.0001)$ also emerged in the older adults' cuneus and lingual gyrus (BA 17/18). These responses were characterized by a greater peak response for rewards than for punishments. Similar engagement of early visual areas has been occasionally noted 


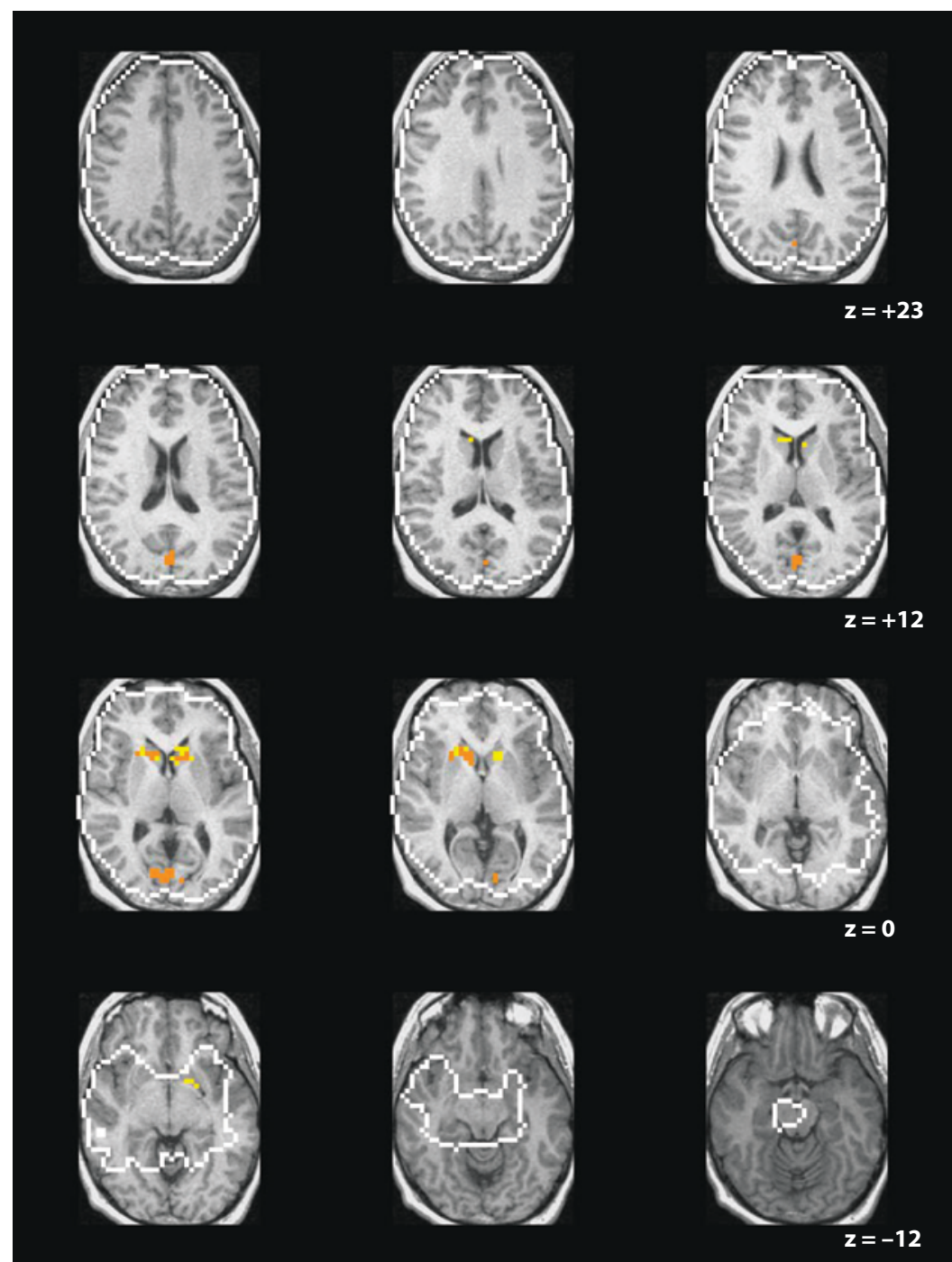

Figure 3. Significant valence $\times$ time interaction effects in the older adults. Note the use of separate significance criteria within and outside of the striatum (see the Method section). Yellow voxels, $p<.001$; orange voxels, $p<.0001$. Clusters also met a contiguity threshold of $\geq 3$ voxels. Images are shown according to radiological convention $($ right $=$ left). The white boundary outlines the masked region that was included in the voxelwise analyses.

Table 1

Valence $\times$ Time Effects in Older Adults

\begin{tabular}{lcccr}
\hline \multicolumn{1}{c}{ Location } & BA & $\begin{array}{c}\text { Peak } \\
F \text { Value }\end{array}$ & $\begin{array}{c}\text { Peak TT } \\
\text { Coordinates }\end{array}$ & $\begin{array}{c}\text { Cluster } \\
\text { Size }\end{array}$ \\
\hline Striatum Mask $(\alpha=.001)$ & & & & \\
$\quad$ Caudate (L) & & 4.74 & $-14,10,8$ & 16 \\
Caudate/putamen (R) & & 5.64 & $13,14,4$ & 24 \\
$\quad$ Putamen (L) & & 3.97 & $-21,0,-4$ & 3 \\
Outside Striatum $(\alpha=.0001)$ & & & & \\
Cuneus/lingual gyrus (L) & $17 / 18$ & 4.59 & $-10,-80,8$ & 3 \\
Cuneus (L/R) & $17 / 18$ & 5.84 & $1,-76,8$ & 23 \\
\hline
\end{tabular}

Note-BA, Brodmann area; TT, Talairach and Tournoux atlas. The laterality of each effect is indicated in parentheses. Cluster sizes are reported as number of active voxels. Contiguity threshold is 3 voxels. 
in our prior research with younger adults (Delgado et al., 2003; Tricomi et al., 2004; Tricomi et al., 2006), but the significance of this outcome activity is not yet clear. The interaction effects could simply reflect discrimination of the outcome display features (e.g., color; see Engel, Zhang, \& Wandell, 1997), or they may indicate affective modulation of the visual cortex, as has been previously shown using emotional face stimuli (Pessoa, McKenna, Gutierrez, \& Ungerleider, 2002).

Striatal time-course features in the older adults. Hemodynamic time courses were plotted for the three valence $\times$ time clusters that fell within the striatum mask (Figure 4). Within the right caudate/putamen, left caudate, and left ventral putamen, the older adults demonstrated the generally expected pattern of striatal activation, with a reward response that was sustained relative to the response observed for punishment. The $t$ tests performed at timepoints T7 and T8 (6-9 sec postoutcome) confirmed that older adults' reward and punishment responses did significantly differ at these timepoints $(p<.002$ for both timepoints and for all three regions).

The other typical feature of the striatal responseinitially greater activity for punishment versus reward outcomes - was not reliably present in the older adults' time courses. The $t$ tests that focused on our early time period of interest (T4, 1.5-3.0 sec postoutcome) did not show any significant divergence of the punishment and reward responses ( $p>.44$ for the three striatal clusters).
Similarly, although some trends suggested that the early punishment feature might appear at a slight delay, $t$ tests on the T5 timepoint (3.0-4.5 sec postoutcome) were also nonsignificant ( $p>.09$ for all clusters). Thus, although the expected late time-course features were clearly replicated in the older adults, the early differentiation between the punishment and reward signals was not robustly demonstrated in this sample.

\section{Between-Groups Comparison}

Overlay of the older and younger adults' outcome activity. As anticipated, the present younger adult sample also replicated the expected focus of striatal outcome activity (Figure 5). Overlay of the younger and older adults' valence $\times$ time effects identified two overlapping striatal regions, including clusters in the right caudate/putamen ( size $=13$ voxels) and the left caudate (size $=7$ voxels). Outside of these two conjunction regions, reliable valence $\times$ time effects appeared to be generally more extensive in the younger participants; this trend is further considered in the voxelwise analyses.

Comparison of the older and younger adults' striatal time courses. Primary time-course analyses focused on the left and right conjunction clusters. To permit better visualization of possible aging effects, we directly superimposed the older and younger adults' time courses for each valence (Figure 6). Relative to those of the older adults, the younger adults' responses appeared to express
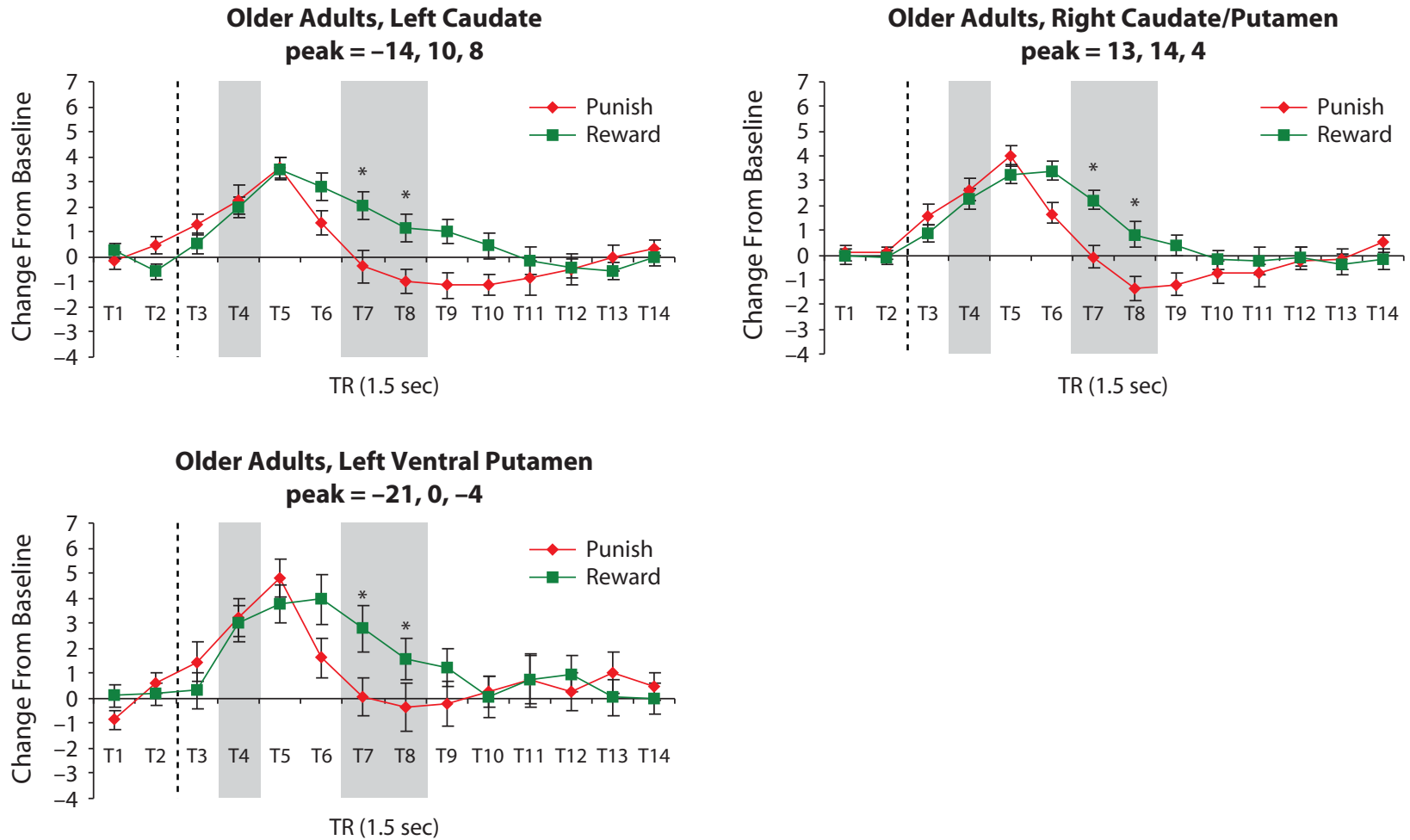

Figure 4. Hemodynamic time courses extracted from the three striatal clusters that showed significant valence $\times$ time effects in the older adults. Dashed line indicates onset of the outcome display, and shaded regions highlight a priori timepoints of interest. Asterisks denote significant differences between the reward and punishment means at a specific timepoint; $p<.05$, corrected. For purposes of presentation, a preoutcome baseline estimate was subtracted from the single-participant time courses prior to plotting the group averages [preoutcome baseline = mean(reward T1, reward T2, punish T1, punish T2)]. Error bars represent standard errors of the means. 


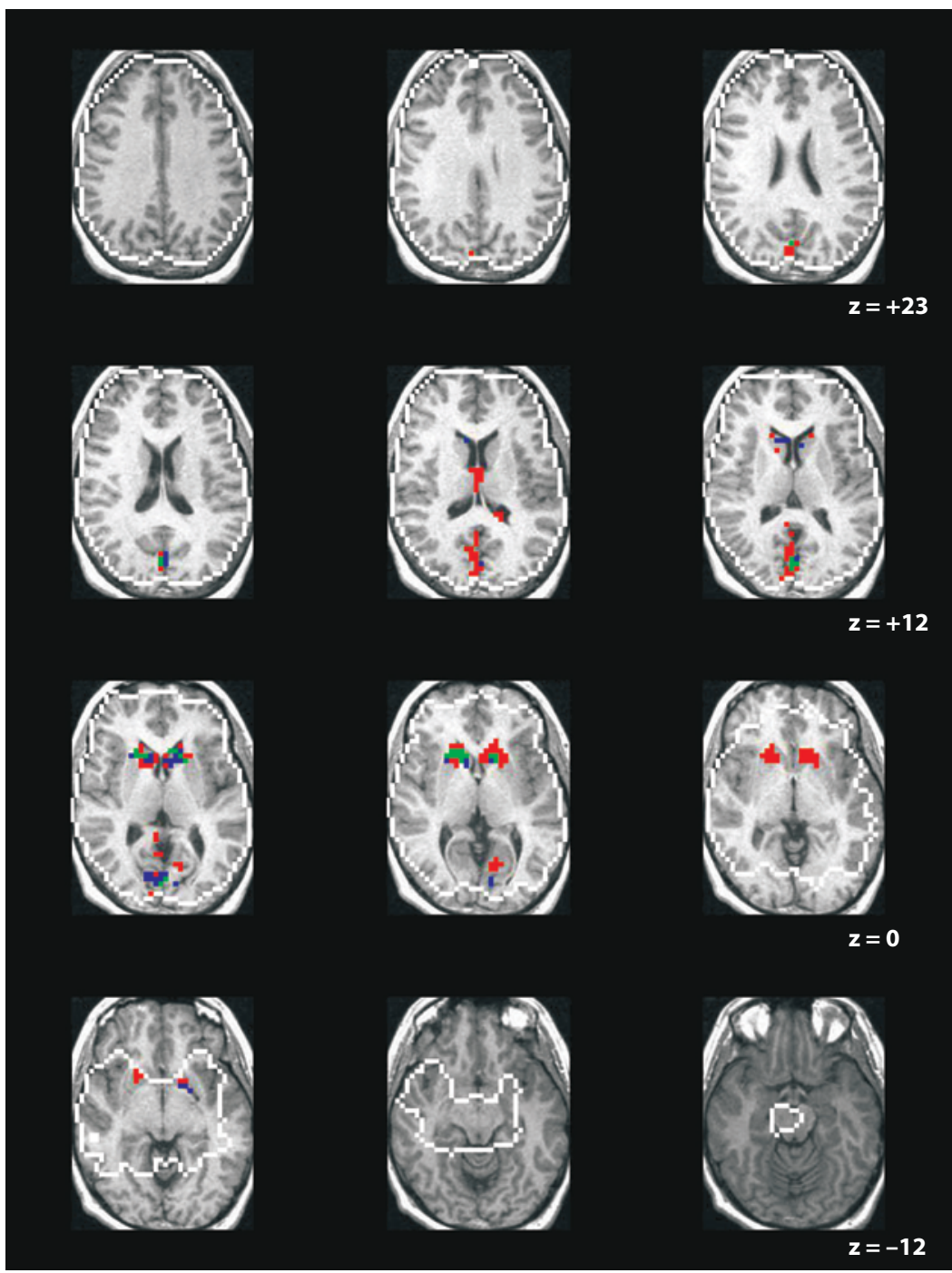

Figure 5. Overlay of the older and younger adults' statistical maps for the valence $\times$ time interaction. As in Figure 3, separate thresholds were used within $(p<.001)$ and outside $(p<.0001)$ the striatum; clusters also met a contiguity threshold of $\geq 3$ voxels. Blue voxels $=$ active in older adults only, red voxels $=$ active in younger adults only, green voxels $=$ active in both older and younger adults. Images are shown according to radiological convention $($ right $=$ left). The white boundary outlines the masked region that was included in the voxelwise analyses.

a generally greater dynamic range and wider differentiation between the two outcome types, although these trends were more notable in the large right cluster than in the smaller left cluster. Quantitative comparisons of the two groups' response profiles (including both reward and punishment, T3-T14) were consistent with this impression. Although no reliable group-level interactions were found in the left cluster [group $\times$ time, $F(11,341)=1.01$, $p=.44$; group $\times$ valence $\times$ time, $F(11,341)=1.35, p=$ .19], the right cluster did exhibit marginally significant group $\times$ time $[F(11,341)=1.74, p=.065]$ and group $\times$ valence $\times$ time $[F(11,341)=1.72, p=.068]$ effects.

Although the marginal and unilateral status of these interactions precludes any strong statements regarding older adults' outcome signaling, their presence in our somewhat limited sample (which may be prone to Type II error) suggests that they merit further exploration. The three-way group $\times$ valence $\times$ time interaction was of particular interest, because it implied a possible difference in the two groups' relative signaling of reward and punishment. Thus, in order to help identify the specific time windows that may have contributed to the marginal three-way trend, a group $\times$ valence ANOVA was performed at each of the 12 postoutcome timepoints. At a Bonferroni-corrected threshold of $p<.05$ (i.e., an effective threshold of $p<.004)$, no reliable group $\times$ valence effects were present. At an uncorrected threshold $(p<.05)$, a single effect was present at timepoint $\mathrm{T} 4[F(1,31)=5.08$, $p=.031]$. Consistent with our earlier tests of the older adults' time-course features, the older participants showed little difference between the early punishment and reward responses, 

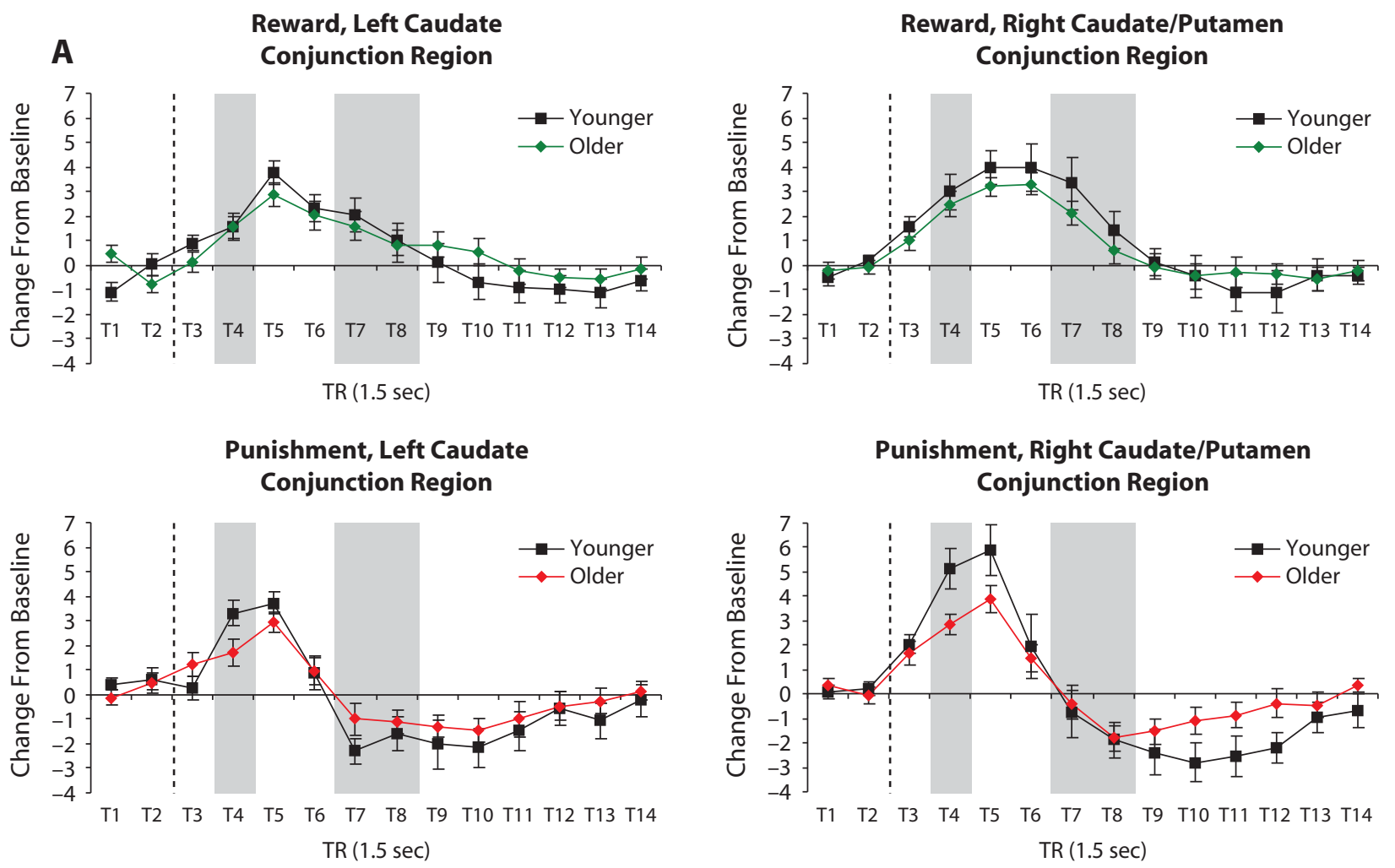

B Reward, Left Caudate Group $\times$ Time Cluster
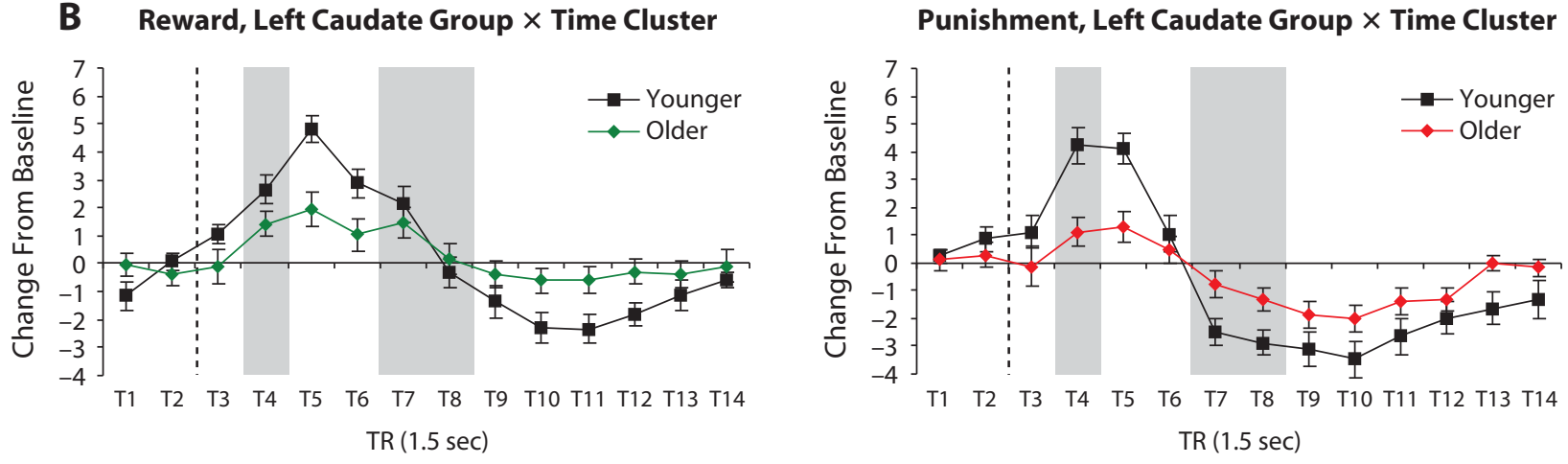

Figure 6. (A) Direct overlay of the older and younger adults' average striatal responses for the reward and punishment trials, respectively. Note that time courses were derived from the left and right striatal voxels that exhibited significant valence $\times$ time effects in both age groups (see green voxels, Figure 5). The dashed line indicates onset of the outcome display, and shaded regions highlight a priori timepoints of interest. For purposes of presentation, a preoutcome baseline estimate was subtracted from the plotted time courses (for details, see Figure 4). Error bars represent standard errors of the means. (B) Example of age-related blunting effects in the left caudate. Time-course presentation follows the same conventions as were used in panel $\mathrm{A}$.

whereas a larger, reliable difference was seen in the younger participants [punishment $>$ reward, $t(12)=-3.96, p=$ .002]. Inspection of the time-course plots (Figure 6) suggested that the loss of the early valence differentiation in the older adults was due to a decrease in the punishment signal, as opposed to an increase in the reward response.

Voxelwise comparisons of the older and younger adults. An additional comparison of the two groups' response profiles was conducted on a voxelwise basis. At our predetermined thresholds, no significant group $\times$ valence $\times$ time effects were found either within or outside of the striatum mask. This absence of strong three-way effects is perhaps not surprising, given the statistical power required for establishing higher order interactions at the single-voxel level. Within the striatum, relaxation of the statistical threshold did not reveal any group $\times$ valence $\times$ time effects until a 3-voxel cluster (peak $=-21,-3,4)$ emerged at $p<.01$. This posterior putamen cluster appeared unlikely to represent a meaningful effect, given its location (outside of either group's outcome-sensitive regions) and the associated time-course patterns (which lacked the expected response features). 
Table 2

Group $\times$ Time Effects

\begin{tabular}{lcccr}
\hline \multicolumn{1}{c}{ Location } & Peak & $\begin{array}{c}\text { Peak TT } \\
\text { Coordinates }\end{array}$ & $\begin{array}{r}\text { Cluster } \\
\text { Size }\end{array}$ \\
\hline Striatum Mask $(\alpha=.001)$ & & & & \\
$\quad$ Caudate (L) & & 7.79 & $-3,10,4$ & 20 \\
$\quad$ Caudate (R) & & 3.99 & $9,18,12$ & 6 \\
$\quad$ Putamen (R) & 3.60 & $16,14,0$ & 3 \\
Outside Striatum $(\alpha=.0001)$ & & & \\
$\quad$ Frontal cortex & & & \\
$\quad$ Precentral gyrus (R) & $4 / 6$ & 5.26 & $47,-3,54$ & 5 \\
$\quad$ Precentral gyrus (R) & $4 / 6$ & 4.39 & $32,-10,54$ & 3 \\
$\quad$ Medial frontal gyrus/cingulate gyrus (L/R) & $6 / 32$ & 6.66 & $1,10,46$ & 64 \\
$\quad$ Inferior frontal gyrus (R) & 47 & 5.40 & $51,14,0$ & 4 \\
Insular cortex & & & & \\
$\quad$ Insula (L) & & 5.61 & $-25,25,8$ & 5 \\
$\quad$ Insula (R) & & 4.14 & $36,25,12$ & 3 \\
Parietal cortex & & & & \\
$\quad$ Postcentral gyrus (R) & 3 & 4.03 & $47,-16,39$ & 4 \\
$\quad$ Precuneus (R) & 7 & 4.74 & $9,-72,42$ & 8 \\
$\quad$ Occipital cortex & & & & \\
$\quad$ Cuneus (R) & 19 & 5.81 & $1,-72,31$ & 9 \\
$\quad$ Cuneus (R) & 18 & 4.84 & $13,-76,15$ & 7 \\
\hline
\end{tabular}

Note-BA, Brodmann area; TT, Talairach and Tournoux atlas. The laterality of each effect is indicated in parentheses. Cluster sizes are reported as number of active voxels. Contiguity threshold is 3 voxels.

Voxelwise tests of the group $\times$ time interaction did yield three significant striatal clusters $(p<.001)$, including a left caudate region ( peak $=-3,10,4$; cluster size $=$ 20 voxels), a small region bordering on the right ventral putamen $($ peak $=16,14,0 ;$ size $=3$ voxels $)$, and another small region bordering on the right caudate/nearby lateral ventricle (peak $=9,18,12$; size $=6$ voxels). Noisy time-course patterns in the right caudate/ventricle cluster pointed to a potentially artifactual cause for the group $\times$ time effects in this region (e.g., coregistration error created by ventricular enlargement). The left caudate and right putamen effects appeared to be of greater relevance, since both of these clusters overlapped with voxels that had shown valence $\times$ time effects in the younger group, but not in the older group (see Figure 5, red voxels). The caudate and putamen group $\times$ time clusters might therefore be interpreted as indicating a lesser extent of striatal recruitment in the older group. Time courses from these regions were generally flatter in the older than in the younger adults, as would be expected if the group $\times$ time effects represented the fringes of the older participants' activation (see left caudate example, Figure 6).

Outside of the striatum, the voxelwise analyses identified 10 cortical regions with significant group $\times$ time effects $(p<.0001$; Table 2$)$. As in the striatum, all of these additional group $\times$ time effects reflected an age-related response blunting: Relative to the younger adults' activating or deactivating trends, the older adults' activity was either flat or reduced. In contrast with the striatal clusters, the cortical clusters were located in voxels that were not associated with significant valence $\times$ time effects in either age group. Overall, then, the data appear to indicate a general decline in the older adults' responsiveness, so that decreased activity was not restricted to the regions that were reliably involved in signaling outcome valence.

\section{DISCUSSION}

The present study addressed two goals: (1) to determine whether the major features of striatal outcome activity are retained in healthy older adulthood, and (2) to explore the possibility of age-related differences in striatal outcome signaling. The relevant findings for each of these questions are discussed in turn.

The primary contribution of our data is the confirmation that older adults do express most of the major features of striatal outcome activity. On the basis of extensive younger adult data from an instrumental paradigm, we specified a set of properties that appear to be typical of the striatal response. Two of these properties were reliably present in our older adult sample. First, the older adults retained the anticipated anatomical focus of outcome sensitivity, so that the caudate head emerged as a key region involved in discriminating between reward and punishment feedback. An additional peak of outcome activity was found in the ventral striatum, as has also been reported in our previous research (Delgado et al., 2000). Second, the dominant temporal component of the outcome response - that is, reliably more sustained activity for rewards than for punishments - was also preserved in the older group, as indicated by significant valence effects during the 6- to 9-sec postoutcome period.

The replication of this activity profile is encouraging, because it identifies reliable response properties that may be targeted in future studies of older adults, using either healthy participants or patient samples. Such studies are likely to be of theoretical relevance, given the existing speculation regarding the mechanisms that drive striatal outcome signaling. Striatal BOLD activation to rewarding versus nonrewarding outcomes is often thought to reflect some form of reward prediction error signal that may be largely generated by midbrain dopamine neurons (see Houk, 
Adams, \& Barto, 1995; Knutson \& Gibbs, 2007). The most compelling evidence for this account has involved the ventral striatum (e.g., O’Doherty, Dayan, Friston, Critchley, \& Dolan, 2003; Pessiglione et al., 2006), although some prediction error models have also been shown to predict dorsal striatal activity (e.g., Haruno \& Kawato, 2006; O’Doherty et al., 2004). Thus, the outcome signals exhibited by our older adults could provide a window onto basic prediction error computations. It is likely, however, that this observed striatal activity also reflects more complex operations. For example, within the majority of the caudate head, outcome sensitivity tends to be restricted to specific scenarios (i.e., instrumental tasks with a perceived action-outcome contingency; see O'Doherty et al., 2004; Tricomi et al., 2004). This context-dependent activity is consistent with the proposed involvement of the caudate in monitoring goal achievement (Tricomi \& Fiez, 2008; Yin \& Knowlton, 2006).

The single striatal response feature that was not clearly replicated in our older participants was the tendency for the punishment response to exceed the reward response during the early postoutcome time window. We cannot exclude the possibility that this feature could be detected with a larger sample or a more powerful design, but it is notable that the early valence differentiation was found in our somewhat smaller younger adult sample. Thus, at minimum, our null results discourage the expectation that the early punishment feature can be reliably observed in a reasonably sized sample of older adults.

A second contribution of this study was to provide a direct comparison of older and younger adults' striatal outcome activity. These analyses were largely motivated by prior research (Eppinger et al., 2008; Samanez-Larkin et al., 2007; Schott et al., 2007) that suggested that the neural processing of rewarding and punishing events may be altered in older adults. The between-groups tests represent the more exploratory component of our study; this work was initially developed to specifically characterize the older adults' response features. However, our group comparisons did identify two trends that indicate some valuable areas for future investigation and caution against prematurely accepting the null hypothesis that striatal outcome processing does not change with age.

First, in a right caudate/putamen region associated with reliable outcome signaling in both age groups, we found trends that indicated a possible change in the older adults' relative signaling of reward and punishment. Exploration of a marginal group $\times$ valence $\times$ time effect in this cluster pointed to a blunted and/or delayed rise in the initial punishment response as a potentially important factor underlying the three-way interaction. This finding should be considered tentative, but it does stress a need for further research into older adults' punishment processing, especially in light of the known declines in their neural reactivity to other kinds of negative stimuli (for punishment-predictive cues, see Samanez-Larkin et al., 2007; for negative feedback, see Eppinger et al., 2008; for negative emotional pictures, see Mather et al., 2004; for negative facial expressions, see Iidaka et al., 2002). Striatal activations to negative outcomes may be an inter- esting target for future aging investigations; recent fMRI studies have documented aversive prediction error signals in the striatum, in addition to the traditional reward prediction error signals (e.g., Seymour et al., 2004; Seymour, Daw, Dayan, Singer, \& Dolan, 2007). Thus, a full understanding of punishment processing in healthy aging may need to address both the activations and deactivations that negative stimuli can evoke in the striatum.

The second aging effect suggested by our data was a generally weaker striatal response in the older adults. In the same caudate/putamen region discussed above, marginally significant group $\times$ time effects pointed to an agerelated reduction in the overall amplitude of striatal activation. Additional patterns suggested a change in the spatial extent of striatal engagement: In the younger adults, robust outcome sensitivity was found in several voxels that did not show significant effects in the older group. Many of these same voxels were associated with a reliable attenuation of the older adults' overall task response.

The present data cannot provide a clear explanation for the older adults' generally reduced responsiveness; this question will need to be addressed by future research. However, one consideration that should be kept in mind is the possibility that either pre- or postoutcome factors may have contributed to this valence-general effect. Since our design did not allow us to completely separate outcome activity from the activation elicited by the preceding choice period, both of these task phases may be relevant to understanding our group $\times$ time effects. For example, it is plausible that reduced anticipatory arousal could partly account for the overall decreased activation seen in the older group. This speculation is supported by our finding of age-related response reductions in the insula, medial frontal cortex (BA 6), and cingulate gyri (BA 32). In addition to the striatum, these cortical regions have also been associated with changes in older adults' anticipatory signaling - reduced reward anticipation in BA 6 and BA 32 (Schott et al., 2007) and reduced loss anticipation in the insula (Samanez-Larkin et al., 2007).

Another factor to consider when interpreting all of our reported results is the relatively young age range of our older adult sample (age range $=51-68$ years). On the basis of the present data, we cannot conclude that the major features of striatal outcome signaling would be preserved in a more elderly group, and we cannot rule out the possibility that more robust or even additional between-groups effects would be found with an older age range. Similarly, given the somewhat older samples included in the Samanez-Larkin et al. (2007) and Schott et al. (2007) studies (age range $=65-81$ and $62-78$ years, respectively), it may be too early to integrate our outcome findings with these authors' anticipation effects. However, it does seem reasonable to expect that our between-groups trends are unlikely to be reversed in an older sample, since the relationship between age and the relevant anatomical variables (e.g., striatal volume and dopamine receptor availability) tends to be monotonic (Bäckman, Nyberg, Lindenberger, Li, \& Farde, 2006; Raz et al., 1995). 
Finally, an important next step for understanding outcome processing in aging is to determine older adults' sensitivity to the magnitude of delivered outcomes. Although we and others (Samanez-Larkin et al., 2007; Schott et al., 2007) have now shown that the aging striatum categorically discriminates between positive and negative outcomes, it is not clear whether older adults also retain the capacity to signal outcome magnitude. Parametric variation of reward and punishment value has been previously shown to modulate younger adults' hemodynamic activity, both within the striatum (Delgado et al., 2003) and in the cortex (for the medial frontal cortex, see Knutson, Fong, Bennett, Adams, \& Hommer, 2003; for the orbitofrontal cortex, see O'Doherty, Kringelbach, Rolls, Hornak, \& Andrews, 2001). Thus, it would be valuable to determine whether such magnitude sensitivity is similarly retained in older adults. Manipulation of gain and loss values may also be useful for detecting differences in older and younger adults' outcome processing, particularly if these effects are selective to the signaling of reward or punishment magnitude.

\section{AUTHOR NOTE}

This research was supported by NIH Grant RO1 DA14103 and DSF Charitable Foundation Grant N023RA02 awarded to the Pittsburgh Institute for Neurodegenerative Diseases. The authors gratefully acknowledge Kate Fissell, Elizabeth Tricomi, Corrine Durisko, and Dave Fraser for technical assistance and helpful discussion. We also thank Minjie Wu and Megan Nable for guidance in implementing the fully deformable coregistration procedure, and the University of Pittsburgh Alzheimer's Disease Research Center (ADRC) for assistance in participant recruitment. Correspondence concerning this article should be addressed to K. M. Cox, Department of Psychology, University of Pittsburgh, 613 LRDC, 3939 O’Hara Street, Pittsburgh, PA 15260 (e-mail:kmc51@pitt.edu).

\section{REFERENCES}

Aizenstein, H. J., Clark, K. A., Butters, M. A., Cochran, J., Stenger, V. A., Meltzer, C. C., ET Al. (2004). The BOLD hemodynamic response in healthy aging. Journal of Cognitive Neuroscience, 16, 786-793.

Bäckman, L., Ginovart, N., Dixon, R. A., Wahlin, T.-B., Wahlin, Å., Halldin, C., \& FARDE, L. (2000). Age-related cognitive deficits mediated by changes in the striatal dopamine system. American Journal of Psychiatry, 157, 635-637.

Bäckman, L., Nyberg, L., Lindenberger, U., Li, S.-C., \& FArde, L. (2006). The correlative triad among aging, dopamine, and cognition: Current status and future prospects. Neuroscience \& Biobehavioral Reviews, 30, 791-807.

Beck, A. T., Steer, R. A., \& Brown, G. K. (1996). Manual for the Beck Depression Inventory (2nd ed.). San Antonio, TX: Psychological Corporation.

Berns, G. S., McClure, S. M., Pagnoni, G., \& Montague, P. R. (2001). Predictability modulates human brain response to reward. Journal of Neuroscience, 21, 2793-2798.

Carstensen, L. L., Pasupathi, M., Mayr, U., \& Nesselroade, J. R. (2000). Emotional experience in everyday life across the adult life span. Journal of Personality \& Social Psychology, 79, 644-655.

Cox, R. W. (1996). AFNI: Software for analysis and visualization of functional magnetic resonance neuroimages. Computers \& Biomedical Research, 29, 162-173.

Delgado, M. R., Locke, H. M., Stenger, V. A., \& Fiez, J. A. (2003). Dorsal striatum responses to reward and punishment: Effects of valence and magnitude manipulations. Cognitive, Affective, \& Behavioral Neuroscience, 3, 27-38.

Delgado, M. R., Miller, M. M., Inati, S., \& Phelps, E. A. (2005). An fMRI study of reward-related probability learning. Neurolmage, 24, 862-873.
Delgado, M. R., Nystrom, L. E., Fissell, C., Noll, D. C., \& Fiez, J. A. (2000). Tracking the hemodynamic responses to reward and punishment in the striatum. Journal of Neurophysiology, 84, 3072-3077.

Delgado, M. R., Stenger, V. A., \& Fiez, J. A. (2004). Motivationdependent responses in the human caudate nucleus. Cerebral Cortex, 14, 1022-1030.

Denburg, N. L., Recknor, E. C., Bechara, A., \& Tranel, D. (2006). Psychophysiological anticipation of positive outcomes promotes advantageous decision-making in normal older persons. International Journal of Psychophysiology, 61, 19-25.

ENGEL, S., Zhang, X., \& WANDELl, B. (1997). Colour tuning in human visual cortex measured with functional magnetic resonance imaging. Nature, 388, 68-71

Eppinger, B., Kray, J., Mock, B., \& Mecklinger, A. (2008). Better or worse than expected? Aging, learning, and the ERN. Neuropsychologia, 46, 521-539.

Folstein, M. F., Folstein, S. E., \& McHugh, P. R. (1975). “Mini-mental state": A practical method for grading the cognitive state of patients for the clinician. Journal of Psychiatric Research, 12, 189-198.

Forman, S. D., Cohen, J. D., Fitzgerald, M., Eddy, W. F., MinTUN, M. A., \& NoLL, D. C. (1995). Improved assessment of significant activation in functional magnetic resonance imaging (fMRI): Use of a cluster-size threshold. Magnetic Resonance in Medicine, 33, 636-647.

Friston, K. J., Ashburner, J., Frith, C. D., Poline, J.-B., Heather, J. D., \& FrackowIAK, R. S. J. (1995). Spatial registration and normalization of images. Human Brain Mapping, 3, 165-189.

Haruno, M., \& Kawato, M. (2006). Different neural correlates of reward expectation and reward expectation error in the putamen and caudate nucleus during stimulus-action-reward association learning. Journal of Neurophysiology, 95, 948-959.

Hasan, K. M., Halphen, C., Boska, M. D., \& Narayana, P. A. (2008). Diffusion tensor metrics, T2 relaxation, and volumetry of the naturally aging human caudate nuclei in healthy young and middle-aged adults: Possible implications for the neurobiology of human brain aging and disease. Magnetic Resonance in Medicine, 59, 7-13.

Hollerman, J. R., \& Schultz, W. (1998). Dopamine neurons report an error in the temporal prediction of reward during learning. Nature Neuroscience, 1, 304-309.

Houk, J. C., Adams, J. L., \& Barto, A. G. (1995). A model of how the basal ganglia generate and use neural signals that predict reinforcement. In J. C. Houk, J. L. Davis, \& D. G. Beiser (Eds.), Models of information processing in the basal ganglia (pp. 249-270). Cambridge, MA: MIT Press.

Iidaka, T., OKada, T., Murata, T., Omori, M., Kosaka, H., Sadato, N., \& YoneKura, Y. (2002). Age-related differences in the medial temporal lobe responses to emotional faces as revealed by fMRI. Hippocampus, 12, 352-362.

Knutson, B., Fong, G. W., Bennett, S. M., Adams, C. M., \& HomMER, D. (2003). A region of mesial prefrontal cortex tracks monetarily rewarding outcomes: Characterization with rapid event-related fMRI. Neurolmage, 18, 263-272.

KnUtson, B., \& GibBs, S. E. B. (2007). Linking nucleus accumbens dopamine and blood oxygenation. Psychopharmacology, 191, 813-822.

Knutson, B., Westdorp, A., Kaiser, E., \& Hommer, D. (2000). fMRI visualization of brain activity during a monetary incentive delay task. NeuroImage, 12, 20-27.

Lipschutz, B., Friston, K. J., Ashburner, J., Turner, R., \& Price, C. J. (2001). Assessing study-specific regional variations in fMRI signal. Neurolmage, 13, 392-398.

Marschner, A., Mell, T., Wartenburger, I., Villringer, A., ReiSCHIES, F. M., \& HeEKEREN, H. R. (2005). Reward-based decisionmaking and aging. Brain Research Bulletin, 67, 382-390.

Mather, M., Canli, T., English, T., Whitfield, S., Wais, P., Ochsner, K., ET AL. (2004). Amygdala responses to emotionally valenced stimuli in older and younger adults. Psychological Science, 15, 259-263.

Mather, M., \& Carstensen, L. L. (2005). Aging and motivated cognition: The positivity effect in attention and memory. Trends in Cognitive Sciences, 9, 496-502.

Mell, T., Heekeren, H. R., Marschner, A., Wartenburger, I., VillRINGER, A., \& ReISchiEs, F. M. (2005). Effect of aging on stimulusreward association learning. Neuropsychologia, 43, 554-563.

Nieuwenhuis, S., Heslenfeld, D. J., Alting von Geusau, N. J., 
Mars, R. B., Holroyd, C. B., \& Yeung, N. (2005). Activity in human reward-sensitive brain areas is strongly context dependent. NeuroImage, 25, 1302-1309.

O’Doherty, J. P., Dayan, P., Friston, K., Critchley, H., \& Dolan, R. J. (2003). Temporal difference models and reward-related learning in the human brain. Neuron, 38, 329-337.

O'Doherty, J. [P.], Dayan, P., Schultz, J., Deichmann, R., FrisTON, K., \& Dolan, R. J. (2004). Dissociable roles of ventral and dorsal striatum in instrumental conditioning. Science, 304, 452-454.

O’Doherty, J. [P.], Kringelbach, M. L., Rolls, E. T., HornaK, J., \& ANDREws, C. (2001). Abstract reward and punishment representations in the human orbitofrontal cortex. Nature Neuroscience, 4, 95-102.

Pessiglione, M., Seymour, B., Flandin, G., Dolan, R. J., \& Frith, C. D. (2006). Dopamine-dependent prediction errors underpin reward-seeking behaviour in humans. Nature, 442, 1042-1045.

Pessoa, L., McKenna, M., Gutierrez, E., \& Ungerleider, L. G. (2002). Neural processing of emotional faces requires attention. Proceedings of the National Academy of Sciences, 99, 11458-11463.

Ravel, S., Legallet, E., \& Apicella, P. (2003). Responses of tonically active neurons in the monkey striatum discriminate between motivationally opposing stimuli. Journal of Neuroscience, 23, 8489-8497.

Raz, N., Gunning-Dixon, F., Head, D., Rodrigue, K. M., WilliamSON, A., \& ACKER, J. D. (2004). Aging, sexual dimorphism, and hemispheric asymmetry of the cerebral cortex: Replicability of regional differences in volume. Neurobiology of Aging, 25, 377-396.

Raz, N., Torres, I. J., \& ACKER, J. D. (1995). Age, gender, and hemispheric differences in human striatum: A quantitative review and new data from in vivo MRI morphometry. Neurobiology of Learning \& Memory, 63, 133-142.

Richter, W., \& Richter, M. (2003). The shape of the fMRI BOLD response in children and adults changes systematically with age. NeuroImage, 20, 1122-1131.

Roitman, M. F., Wheeler, R. A., \& Carelli, R. M. (2005). Nucleus accumbens neurons are innately tuned for rewarding and aversive taste stimuli, encode their predictors, and are linked to motor output. Neuron, 45, 587-597.

Samanez-Larkin, G. R., Gibbs, S. E. B., Khanna, K., Nielsen, L., Carstensen, L. L., \& KnUtson, B. (2007). Anticipation of monetary gain but not loss in healthy older adults. Nature Neuroscience, 10, 787-791.

Schneider, W., Eschman, A., \& Zuccolotto, A. (2002). E-Prime reference guide. Pittsburgh, PA: Psychology Software Tools.

Schott, B. H., Niehaus, L., Wittmann, B. C., Schütze, H., SeidenBeCher, C. I., HeInze, H.-J., \& Düzel, E. (2007). Ageing and earlystage Parkinson's disease affect separable neural mechanisms of mesolimbic reward processing. Brain, 130, 2412-2424.

Schultz, W., Tremblay, L., \& Hollerman, J. R. (2000). Reward processing in primate orbitofrontal cortex and basal ganglia. Cerebral Cortex, 10, 272-283.

Seymour, B., Daw, N., Dayan, P., Singer, T., \& Dolan, R. (2007). Differential encoding of losses and gains in the human striatum. Journal of Neuroscience, 27, 4826-4831.

Seymour, B., O’Doherty, J. P., Dayan, P., Koltzenburg, M., Jones, A. K., Dolan, R. J., ET AL. (2004). Temporal difference models describe higher-order learning in humans. Nature, 429, 664-667.

Swainson, R., SenGupta, D., Shetty, T., Watkins, L. H. A., SumMERS, B. A., SAHAKIAN, B. J., ET AL. (2006). Impaired dimensional selection but intact use of reward feedback during visual discrimination learning in Parkinson's disease. Neuropsychologia, 44, 1290-1304.

TAlairach, J., \& Tournoux, P. (1988). Co-planar stereotaxic atlas of the human brain: 3-Dimensional proportional system-An approach to cerebral imaging. Stuttgart: Thieme Medical Publishers.

Tricomi, E., Delgado, M. R., \& Fiez, J. A. (2004). Modulation of caudate activity by action contingency. Neuron, 41, 281-292.

Tricomi, E., Delgado, M. R., McCandliss, B. D., McClelland, J. L., \& FieZ, J. A. (2006). Performance feedback drives caudate activation in a phonological learning task. Journal of Cognitive Neuroscience, 18, 1029-1043.
Tricomi, E., \& FiEZ, J. A. (2008). Feedback signals in the caudate reflect goal achievement on a declarative memory task. NeuroImage, 41, 1154-1167.

Tversky, A., \& Kahneman, D. (1981). The framing of decisions and the psychology of choice. Science, 211, 453-458.

Ungless, M. A., Magill, P. J., \& Bolam, J. P. (2004). Uniform inhibition of dopamine neurons in the ventral tegmental area by aversive stimuli. Science, 303, 2040-2042.

Volkow, N. D., Gur, R. C., WAng, G.-J., Fowler, J. S., MoberG, P. J., DING, Y.-S., ET AL. (1998). Association between decline in brain dopamine activity with age and cognitive and motor impairment in healthy individuals. American Journal of Psychiatry, 155, 344-349.

Wang, Y., Chan, G. L. Y., Holden, J. E., Dobko, T., MaK, E., Schulzer, M., ET AL. (1998). Age-dependent decline of dopamine D1 receptors in human brain: A PET study. Synapse, 30, 56-61.

WARD, B. D. (2001). Deconvolution analysis of $f M R I$ time series data. Milwaukee: Medical College of Wisconsin, Biophysics Research Institute.

Weiler, J. A., Bellebaum, C., \& Daum, I. (2008). Aging affects acquisition and reversal of reward-based associative learning. Learning \& Memory, 15, 190-197.

Williams, Z. M., \& Eskandar, E. N. (2006). Selective enhancement of associative learning by microstimulation of the anterior caudate. Nature Neuroscience, 9, 562-568.

Wittmann, B. C., Schott, B. H., Guderian, S., Frey, J. U., Heinze, H.-J., \& DüZEL, E. (2005). Reward-related fMRI activation of dopaminergic midbrain is associated with enhanced hippocampus-dependent longterm memory formation. Neuron, 45, 459-467.

Woods, R. P., Grafton, S. T., Holmes, C. J., Cherry, S. R., \& MazzIOTTA, J. C. (1998). Automated image registration: I. General methods and intrasubject, intramodality validation. Journal of Computer Assisted Tomography, 22, 139-152.

Woods, R. P., Grafton, S. T., Watson, J. D. G., Sicotte, N. L., \& Mazziotta, J. C. (1998). Automated image registration: II. Intersubject validation of linear and nonlinear models. Journal of Computer Assisted Tomography, 22, 153-165.

Wu, M., Carmichael, O., Lopez-Garcia, P., Carter, C. S., \& AizenSTEIN, H. J. (2006). Quantitative comparison of AIR, SPM, and the fully deformable model for atlas-based segmentation of functional and structural MR images. Human Brain Mapping, 27, 747-754.

Wu, M., Mazurkewicz, L. M., Nable, M., \& Aizenstein, H. J. (2006, October). Improved fMRI coregistration with a fully deformable model. Poster presented at the 36th Annual Meeting of the Society for Neuroscience, Atlanta.

YIN, H. H., \& KNOWLTON, B. J. (2006). The role of the basal ganglia in habit formation. Nature Reviews Neuroscience, 7, 464-476.

\section{NOTES}

1. The fully deformable registration procedure was one of multiple candidate methods we considered for colocalization of the functional data. Other options included the 12-parameter affine option of the AIR "alignlinear" program (Woods, Grafton, Watson, et al., 1998) and manual tracing of anatomically defined striatal regions of interest (ROIs). These two alternatives yielded results that were generally comparable to those observed for the fully deformable registration, although the linear registration and ROI methods tended to generate less robust functional effects.

2. To further confirm that trial-based averaging was appropriate for this study, we conducted a second analysis, in which the full jittered data set was submitted to the AFNI 3dDeconvolve program (Ward, 2001). Hemodynamic responses were modeled for the first $24 \mathrm{sec}$ (16 scans) following trial onset. No assumptions of response shape were made. Time courses obtained with this deconvolution approach were similar to those estimated with trial-based averaging.

(Manuscript received December 22, 2007; revision accepted for publication June 7, 2008.) 